\title{
A Breakdown in the Special Theory of Relativity Demonstrated Based on an Elucidation of the Relativity of Time
}

\author{
Koshun Suto ${ }^{1}$ \\ ${ }^{1}$ Chudaiji Buddhist Temple, Isesaki, Japan \\ Correspondence: Koshun Suto, Chudaiji Buddhist Temple, 5-24, Oote-Town, Isesaki, 372-0048, Japan. \\ Tel: 81-270-23-9980. E-mail: koshun_suto129@mbr.nifty.com
}

Received: June 8, 2018

Accepted: June 27, 2018

Online Published: July 14, 2018

doi:10.5539/apr.v10n4p38

URL: https://doi.org/10.5539/apr.v10n4p38

\begin{abstract}
In the thought experiment in this paper, we consider inertial frames $\mathrm{M}$ and $\mathrm{A}$ moving at constant velocity relative to each other. First, a light signal is emitted from inertial frame $M$ toward inertial frame A when the time on a clock in inertial frame $\mathrm{M}$ is 1 (s). In the scenario of this paper, that light arrives at inertial frame $\mathrm{A}$ when time on the clock in A is 2 (s). Next, the situation is reversed, and a light signal is emitted from inertial frame A toward inertial frame $\mathrm{M}$ when the time in inertial frame $\mathrm{A}$ is 1 (s). That light arrives at inertial frame $\mathrm{M}$ when the time in $\mathrm{M}$ is 2 (s). According to the special theory of relativity (STR), the two inertial frames are equivalent, and thus it is not surprising that symmetric experiment results are obtained. However, it has already been pointed out that, among the coordinate systems regarded by Einstein as inertial frames, there are "classically stationary frames" where light propagates isotropically, and "classically moving frames" where light propagates anisotropically. If a classically stationary frame is incorporated into a thought experiment, it becomes easier to predict the experiment results. This paper elucidates a system whereby symmetrical experiment results can be obtained, even if the two coordinate systems are not equivalent. If one attempts to explain such experiment results from the standpoint of the STR, it ironically requires the use of logic that is unacceptable under the STR. Thus, this paper explains those experiment results by using logic different from the STR, and demonstrates the breakdown in the STR.
\end{abstract}

Keywords: Special Theory of Relativity; Classically Stationary Frames; Relativistically Stationary Frames; Classically Stationary Frames; Minkowski Diagram; Velocity Vector

\section{Introduction}

As a physical theory representing the 20th century, Einstein's special theory of relativity has held sway in the world of physics for more than a century. During this time, the STR has fended off challenges and counterarguments from many physicists.

The STR is not just a single theoretical system. It is composed of two theories of different types. The first is a theory derived from Lorentz transformations which has full symmetry, and the second is Einstein's energymomentum relationship which holds in free space.

Of these two theories, it is the former that is treated as a problem in this paper.

Now, consider a situation where two rods, which have the same length when stationary, are moving at constant velocity along the $x$-axis. Clocks of the same type are placed on the two rods, rod I and rod II. Here, the coordinate system of rod I is taken to be frame $S$, and the coordinate system of rod II to be frame $S^{\prime}$. The relative velocity of frame $S$ and frame $S^{\prime}$ is taken to be $v$.

According to the STR, when an observer in frame $S$ measures the time which elapses on the clock in frame $S^{\prime}$, the time which elapses in frame $S^{\prime}$ is delayed compared to the time which elapses in frame $S$. Next, the observer in frame $S^{\prime}$ measures the length of rod I placed on the $x$-axis in frame $S$ by using a clock that is advancing slowly in frame $S^{\prime}$. The observer in frame $S^{\prime}$ measures the time $t$ required for both ends of rod I to pass in front of himself, and finds the rod length $v t$. At this time, rod I is shorter than rod II. Next, when the situation is reversed, and the observer in frame $S^{\prime}$ makes observations, the time which elapses on the clock in frame $S$ is delayed. Next, when the observer in frame $S$ measures the length of rod II in frame $S^{\prime}$ using a clock which is slowly advancing in frame $S$, the rod is contracted in the direction of motion. According to the "principle of 
relativity," the two inertial frames are equivalent, and thus the observers in frame $S$ and frame $S^{\prime}$ measure the same value as a matter of course.

However, there are problems with the STR. Whereas the delay in time predicted by the STR is a physical delay, the contraction of the rod is not thought to be physical contraction. This is a problem from the standpoint of symmetry. Also, whereas the observer in frame $S$ who observes the delay of time in frame $S^{\prime}$ is an observer in a stationary frame, the clock in frame $S$ where contraction of the rod in frame $S^{\prime}$ is observed is a clock in a slowly advancing moving frame, and this too is a problem.

\section{Problems with the STR}

Two problems with the STR were pointed out in the introduction, but this section discusses an even bigger problem.

\subsection{The "Principle of Constancy of Light Speed E" Created by Einstein}

According to the "principle of relativity" that was assumed when developing the STR, all inertial frames are equivalent. Therefore, the STR denies the existence of inertial frames to which velocity vectors are attached. Einstein developed the STR by asserting that there is no need for the theory to incorporate velocity vectors or the ether (Einstein, 1923).

Einstein assumed the principle of relativity and the "principle of constancy of light speed." The latter includes the following two principles.

"Any ray of light moves in the "stationary" system of co-ordinates with the determined velocity $c$, whether

the ray be emitted by a stationary or by a moving body" (Einstein, 1923).

"Let a ray of light start at the "A time" $t_{\mathrm{A}}$ from A towards B, let it at the "B time" $t_{\mathrm{B}}$ be reflected at B in the direction of $\mathrm{A}$, and arrive again at $\mathrm{A}$ at the "A time" $t_{\mathrm{A}}^{\prime}$.

In agreement with experience we further assume the quantity

$$
\frac{2 \mathrm{AB}}{t_{\mathrm{A}}^{\prime}-t_{\mathrm{A}}}=c
$$

to be a universal constant — the velocity of light in empty space" (Einstein, 1923).

In this paper, we distinguish between the former principle as the "principle of constancy of light speed I" (abbreviated below as "principle I") and the latter principle as the "principle of constancy of light speed II." (abbreviated below as "principle II"). The "principle I" asserts that the light speed in vacuum does not depend on the speed of the light source. The "principle II" asserts that the light speed calculated from the round-trip travel time is constant.

Let there be a given stationary rigid rod of length $L$ as measured by a ruler which is stationary, and assume that the rod is placed along the stationary frame's $x$-axis.

Assume that clocks $\mathrm{A}$ and $\mathrm{B}$ of the same type are set up at points $\mathrm{A}$ and $\mathrm{B}$ on the rear (negative direction) and front (positive direction) end of this rod. Here clock $A$ will be abbreviated as $C_{A}$, and clock $B$ as $C_{B}$.

Suppose a ray of light is emitted in the direction of $\mathrm{B}$ from $\mathrm{A}$ at time $t_{\mathrm{A}}$ of $\mathrm{C}_{\mathrm{A}}$, reaches and is reflected at $\mathrm{B}$ at time $t_{\mathrm{B}}$ of $\mathrm{C}_{\mathrm{B}}$, and then returns to $\mathrm{A}$ at time $t_{\mathrm{A}^{\prime}}$ of $\mathrm{C}_{\mathrm{A}}$. Einstein determined that if the following relationships hold between these two times, then the two clocks represent the same time by definition (Einstein, 1923).

$$
\begin{aligned}
& t_{\mathrm{B}}-t_{\mathrm{A}}=t_{\mathrm{A}^{\prime}}-t_{\mathrm{B}} . \\
& \frac{1}{2}\left(t_{\mathrm{A}}+t_{\mathrm{A}^{\prime}}\right)=t_{\mathrm{B}} .
\end{aligned}
$$

Equations (1) and (2) can also be applied to an inertial frame $S^{\prime}$ in which a rod is moving at constant velocity relative to a stationary frame $S$. (in this case, $t_{\mathrm{A}}$ and $t_{\mathrm{B}}$ become times in frame $S^{\prime}$.)

Now, the rod which was stationary begins to move at constant velocity along the $x$-axis of frame $S$. At an arbitrary time, a light signal is emitted from point $\mathrm{A}$ on the rear side of the rod toward point B on the front side.

If the principle I is applied, then propagation of light in frame $S^{\prime}$ seen from an observer in frame $S$ is anisotropic. Therefore, from the classical perspective, an observer in frame $S^{\prime}$ determines the propagation of light in frame $S^{\prime}$ to be anisotropic in the same way. Also, it is concluded that the speed of light on the outward path and return path is not $c$. 
However, the principle II also holds in frame $S^{\prime}$, and thus the speed of light calculated by the observer in frame $S^{\prime}$ from the round-trip time of the light becomes $c$. However, according to the STR, the two inertial frames are equivalent, and thus the speed of light measured by the observer in frame $S^{\prime}$ must be $c$ for both the outward and return path.

Considered classically, an inertial frame in which light propagates isotropically is a "classically stationary frames $S_{\mathrm{cl}}$," and an inertial frame in which light propagates anisotropically is a "classically moving frames $S_{\mathrm{cl}}^{\prime}$."

However, if two clocks in an inertial frame are synchronized using the method of Einstein, then even in frame $S_{\mathrm{cl}}$, the speed of light is measured as $c$ on both the outward and return path (Relativistic isotropic propagation).

As a result, both frame $S_{\mathrm{cl}}$ and frame $S_{\mathrm{cl}}^{\prime}$ fall under the heading of a "relativistically stationary frames," and it is impossible to experimentally identify the two. Also, all inertial frames become stationary frames in the sense of the principle of relativity.

In this paper, the principle introduced by Einstein is called the "principle of constancy of light speed E." (where "E" stands for Einstein. abbreviated below as "principle E"). That is, Principle of constancy of light speed E: In all inertial frames, light speed of the outward path and return path is constant $(c)$.

The principle II asserts that the light speed calculated from the round-trip travel time is constant.

In contrast, when the times of the clocks at both ends of a rod moving at constant velocity are synchronized using the method of Einstein, then even in a coordinate system where light intrinsically propagates anisotropically, the speed of light is measured as $c$ on both the outward and return paths.

This principle is not a universal principle, but a personal principle introduced by Einstein. To maintain this principle, the observer in a moving frame must adjust the time on a clock each time the velocity of a moving frame changes. If the observer neglects this task, the principle $\mathrm{E}$ is no longer a principle.

For the above reason, it is evident that the shift from classical physics to the STR is achieved through the following manipulations and assumptions.

1) It is assumed that Equation (1) always holds between two clocks placed on the $x$-axis in an inertial frame. (The same holds when the clocks have started moving.)

2) Even if there are coordinate systems, among those regarded by Einstein as inertial frames, which cannot be said to be equivalent from the perspective of light propagation, they are treated as equivalent inertial frames by assuming the principle of relativity.

The above points can be summarized as follows:

$$
\text { Classical physics } \quad \rightarrow \quad \text { STR }
$$

$$
\left[\begin{array}{l}
\text { P rinciple of constancy of lightspeed I } \\
\text { P rinciple of constancy of lightspeed II }
\end{array}\right] \rightarrow \text { Principle of constancy of lightspeed E }
$$

Light signal propagation $\left[\begin{array}{l}\text { A priori isotropic propagation } \\ \text { Anisotropic propagation }\end{array}\right] \rightarrow$ Relativistic isotropic propagation

Inertial frames $\left[\begin{array}{l}\text { Classically stationary frames } S_{\mathrm{cl}} \\ \text { Classically moving frames } S_{\mathrm{cl}}^{\prime}\end{array}\right] \rightarrow$ Relativistically stationary frames $S_{\mathrm{re}}$

According to the principle of relativity, all inertial frames are equivalent. Therefore, the STR does not discriminate between the two types of inertial frames: coordinate systems $S_{\mathrm{cl}}$ in which light propagates isotropically and coordinate systems $S_{\mathrm{cl}}^{\prime}$ in which light propagates anisotropically. Einstein originally recognized that there are two principles of constancy of light speed, principle I and principle II. However, when constructing the STR, Einstein placed higher priority on the principle of relativity than principle I. Also, he proposed that the time of clocks be adjusted so that the speed of light is measured to be $c$ on both the outward and return path. As a result, a new principle E was created. When the STR was constructed, the reason for being of the originally assumed principle I and principle II faded, and their status was usurped by principle E. This paper addresses the case where the STR gives higher priority to principle E than principle I in the thought experiment in section 4.

\subsection{True Factors Determining the Ratio of Space Contraction and Time Dilation}

Next is Einstein's energy-momentum relationship, which holds in free space. 


$$
\left(m c^{2}\right)^{2}=\boldsymbol{p}^{2} c^{2}+\left(m_{0} c^{2}\right)^{2} .
$$

Here, $m_{0} c^{2}$ is the rest mass energy of a particle or object, $m c^{2}$ is the relativistic energy, and $p$ is the momentum. It is known that the following equation can be derived from Equation (1).

$$
m c^{2}=m_{0} c^{2}\left(1-\frac{v^{2}}{c^{2}}\right)^{-1 / 2} .
$$

From this,

$$
\left(1-\frac{v^{2}}{c^{2}}\right)^{1 / 2}=\frac{m_{0}}{m}=\frac{1}{\gamma} .
$$

Now, let us consider two inertial frames $S$ and $S^{\prime}$ which are moving at constant speed relative to each other in free space. Let us assume that the distance between two points $x_{1}$ and $x_{2}$ on the $x$-axis in frame $S$ has been measured as $L$ by an observer in frame $S$. Next, let us assume that the distance between these two points is measured as $L^{\prime}$ by an observer in frame $S^{\prime}$ moving at constant speed $v$ relative to frame $S$. (Here, note that frame $S^{\prime}$ is a stationary frame.)

At this time, the observer in frame $S^{\prime}$ finds the following relationship between $L$ and $L^{\prime}$.

$$
L^{\prime}=L\left(1-\frac{v^{2}}{c^{2}}\right)^{1 / 2} .
$$

The rod in frame $S$ moving at constant velocity with respect to frame $S^{\prime}$ contracts in the direction of motion.

Also, with regard to time, if a time $\tau^{\prime}$ elapses in frame $S^{\prime}$ when a time $\tau$ elapses in frame $S$, then an observer in frame $S^{\prime}$ finds the following relationship between $\tau$ and $\tau^{\prime}$.

$$
\tau^{\prime}=\tau\left(1-\frac{v^{2}}{c^{2}}\right)^{-1 / 2}, \quad \tau<\tau^{\prime} .
$$

The time that elapses on the clock in frame $S$ which is moving at constant velocity with respect to frame $S^{\prime}$ is delayed.

Next, if Equation (5) is substituted into Equations (6) and (7), the following equations are obtained (Suto, 2014).

$$
\begin{aligned}
L^{\prime} & =L \cdot \frac{m_{0}}{m} . \\
\tau^{\prime} & =\tau \cdot \frac{m}{m_{0}} .
\end{aligned}
$$

If an observer in frame $S$ measures the distance $L$ between two points in frame $S^{\prime}$ and takes this to be $L^{\prime}$, then Equation (8) holds between $L$ and $L^{\prime}$. Also, if a time $\tau$ elapses in frame $S^{\prime}$, and this time is measured with a clock in frame $S$ and taken to be $\tau^{\prime}$, then Equation (9) holds between $\tau$ and $\tau^{\prime}$. A point to note here is that Equations (6) and (8), and Equations (7) and (9), are not equivalent. The mass (energy) of a moving object increases as the velocity of the object increases. However, in this case the reverse happens; if the mass of an object of the same type, placed in a stationary frame, is measured from a moving frame, the mass of the object should be smaller. That is, Equations (8) and (9) are the equations when predicting physical quantities of a moving frame from a stationary frame, and cannot be applied in the reverse case. With Equations (8) and (9), inertial frames and moving frames are not equivalent. Therefore, Equations (8) and (9) cannot be regarded as equations of the STR.

The contraction of the rod and delay of time do not depend on the relative velocity of the two coordinate systems. They should be regarded as dependent on the mass (energy) which increases or decreases due to the change in velocity.

\subsection{Method of Discriminating Between a "Classically Stationary Frame" and "Classically Moving Frame"}

Einstein constructed the STR without recognizing the distinction between frames $S_{\mathrm{cl}}$ and $S_{\mathrm{cl}}^{\prime}$. However, at present it is thought to be possible to discriminate between frames $S_{\mathrm{cl}}$ and $S_{\mathrm{cl}}^{\prime}$. 
Let there be a given stationary rigid rod of length $L$ as measured by a ruler which is stationary, and assume that the rod is placed along the classically stationary frame's $x$-axis.

Assume that clocks $\mathrm{A}$ and $\mathrm{B}$ of the same type are set up at points $\mathrm{A}$ and $\mathrm{B}$ on the rear (negative direction) and front (positive direction) end of this rod. Here clock $A$ will be abbreviated as $C_{A}$, and clock $B$ as $C_{B}$.

To begin, synchronization is carried out so that the relationship in Equation (1) holds between the times on these clocks. In this case, the coordinate system of the stationary rod I is frame $S_{\mathrm{cl}}$, and thus the times of the two clocks match in an absolute sense.

Next, this rod begins to move at constant velocity $v$. In this case, the times of the clocks at both ends of the rod must be adjusted so they are simultaneous in the moving frame. Here, the time of $\mathrm{C}_{\mathrm{B}}$ is adjusted (see Figure 1).

Now, if $\Delta t^{\prime}$ is taken to be the adjustment time of $\mathrm{C}_{\mathrm{B}}$ carried out by an observer in a rocket, and the coordinate system in which the rod was initially at rest is the frame $S_{\mathrm{cl}}$, then

$$
\Delta t^{\prime}=\frac{L v}{c^{2}}(\mathrm{~s})
$$

However, if the coordinate system in which the rod was initially at rest is the frame $S_{\mathrm{cl}}^{\prime}$, then

$$
\Delta t^{\prime} \neq \frac{L v}{c^{2}}(\mathrm{~s})
$$

The reason why Equation (10) does not hold in a classically moving frame is because of an unknown velocity vector attached to frame $S_{\mathrm{cl}}^{\prime}$ (Suto, 2010, 2015, 2017a). In the following sections, a thought experiment is carried out while discriminating between frames $S_{\mathrm{cl}}$ and $S_{\mathrm{cl}}^{\prime}$.
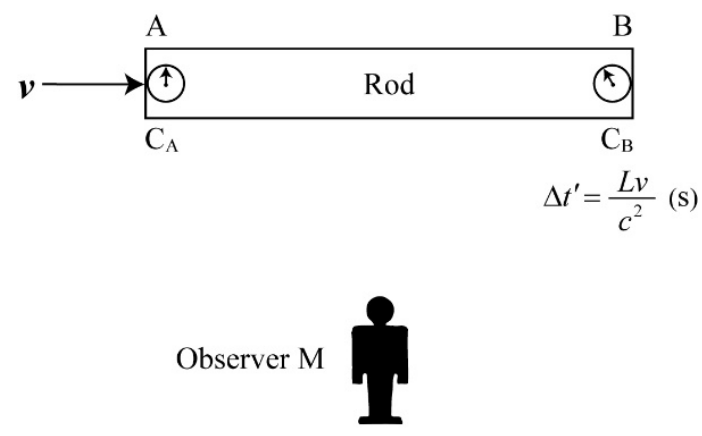

Classically stationary frame

Figure 1. Time adjustment $\Delta t^{\prime}$ of $\mathrm{C}_{\mathrm{B}}$ moving at constant velocity $\boldsymbol{v}$ relative to a "classically stationary frame"

\section{Length Contraction and Time Delation Explainable Using Classical Considerations}

Consider a laboratory whose interior floor is a square. The Michelson interferometer is placed in this laboratory (see Figure 2).

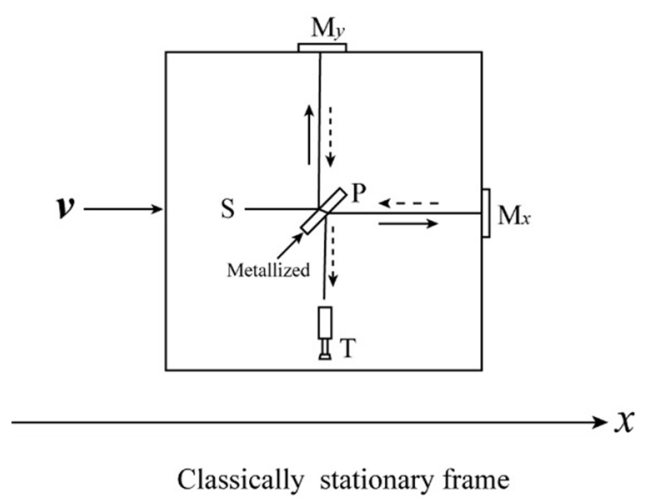

Figure 2. This figure shows the view from above of a laboratory moving at constant velocity with respect to $S_{\mathrm{cl}}$ 
At the center of the room, there is a glass plate (beam splitter) $\mathrm{P}$ with a semi-transparent metal coating on its front face. The angle between this glass plate and the $x$-axis is $45^{\circ}$. Light emitted from the light source $\mathrm{S}$ strikes this glass at an angle, and the light is split in two. One beam passes through the plate, strikes a mirror $\mathrm{M}_{x}$, is reflected, and retraces its path to the splitting point $\mathrm{P}$. On the second light path, the beam is reflected by the glass plate $\mathrm{P}$, arrives at mirror $\mathrm{M}_{y}$, is reflected there, and returns to the splitting point P. (Only the essential parts of the experimental instrument are shown here. Equipment not needed for the discussion in this paper has been omitted.)

This laboratory is moving at constant velocity $\boldsymbol{v}$ along the $x$-axis of frame $S_{\mathrm{cl}}$. The light path length $\mathrm{PM}_{x}$ measured indoors is taken to be $L_{x} / 2$ and the path length $\mathrm{PM}_{y}$, is taken to be $L_{y} / 2$. (However, in measurements in the laboratory, $L_{x}$ and $L_{y}$ are equal.) In addition, the light path length when $L_{x} / 2$ is measured from frame $S_{\mathrm{cl}}$ is taken to be $L_{x}^{\prime} / 2$, and the light path length when $L_{y} / 2$ is measured from frame $S_{\mathrm{cl}}$ is taken to be $L_{y}^{\prime} / 2$. (However, $L_{y}$ and $L_{y}^{\prime}$ are equal.)

Here, the time required for light to make a round trip over $\mathrm{PM}_{x}$ is measured from frame $S_{\mathrm{cl}}$. If this round trip time is taken to be $t_{x}^{\prime}$, then the observer in frame $S_{\mathrm{cl}}$ applies the principle I to this light propagation, and thus:

$$
t_{\mathrm{x}}^{\prime}=\frac{L_{x}^{\prime}}{2(c-v)}+\frac{L_{x}^{\prime}}{2(c+v)}=\frac{L_{x}^{\prime} c}{c^{2}-v^{2}}=\frac{L_{x}^{\prime}}{c\left(1-v^{2} / c^{2}\right)} .
$$

Next, the time for light to make a round trip over $\mathrm{PM}_{y}$ is measured. If this round trip time is measured in frame $S_{\mathrm{cl}}$ and taken to be $t_{y}^{\prime}$, then:

$$
t_{\mathrm{y}}^{\prime}=\frac{L_{y}^{\prime}}{c\left(1-v^{2} / c^{2}\right)^{1 / 2}} .
$$

Here, $t_{x}^{\prime}$ and $t_{y}^{\prime}$ are the times which elapse on the clock in frame $S_{\mathrm{cl}}$.

The method of deriving Equation (13) is explained in many textbooks so here it is omitted.

Incidentally, the predicted effect could not be detected from the Michelson-Morley experiment. This means that $t_{\mathrm{x}}^{\prime}$ and $t_{\mathrm{y}}^{\prime}$ are equal. In the end, the following relationship can be derived from Equations (12) and (13).

$$
L_{x}^{\prime}=\frac{L_{y}^{\prime}}{\gamma} .
$$

Here, $L_{y}^{\prime}$ and $L_{x}$ are equal, so Equation (14) can be written as follows.

$$
L_{x}^{\prime}=\frac{L_{x}}{\gamma} .
$$

When measured from frame $S_{\mathrm{cl}}$, the laboratory contracts by $1 / \gamma$ times in the direction of motion. This contraction is physical contraction due to the fact that some force has acted on the laboratory, and this can be regarded as true contraction (Suto, 2017b).

Incidentally, an observer in the coordinate system $S_{\mathrm{cl}}^{\prime}$ of the laboratory applies the principle II to this light propagation, and thus the round trip times of light $t_{x}$ and $t_{y}$ are predicted as follows:

$$
\begin{aligned}
& t_{\mathrm{x}}=\frac{L_{x}}{c} . \\
& t_{\mathrm{y}}=\frac{L_{y}}{c} .
\end{aligned}
$$

In the end, $t_{y}$ elapses in frame $S_{\mathrm{cl}}^{\prime}$ while $t_{y}^{\prime}$ elapses in frame $S_{\mathrm{cl}}$. In addition, $L_{y}=L_{y}^{\prime}$ and thus Equation (13) can be written as follows:

$$
t_{\mathrm{y}}^{\prime}=\frac{\gamma L_{y}}{c} .
$$

Next, if this is compared with Equations (17) and (18):

$$
t_{\mathrm{y}}^{\prime}=\gamma t_{\mathrm{y}}
$$


When observed from frame $S_{\mathrm{cl}}$, the time $t_{y}$ which elapses in frame $S_{\mathrm{cl}}^{\prime}$ is delayed compared to the time $t_{y}^{\prime}$ which elapses in frame $S_{\mathrm{cl}}$. Actually, this prediction has been verified by experiments where the life of elementary particles is extended. In the end, space contraction and time delay in frame $S_{\mathrm{cl}}^{\prime}$ can be predicted if the principle I and principle II are assumed.

\section{Elucidation of the Relativity of the Passage of Time in Frames $S_{\mathrm{cl}}^{\prime}$ and $S_{\mathrm{cl}}$}

\subsection{Symmetrical Thought Experiments Performed in Frames $S_{\mathrm{cl}}$ and $S_{\mathrm{cl}}^{\prime}$}

Rocket A is moving at constant velocity of $0.6 c$ in the $x$-axis direction of $S_{\mathrm{cl}}$. There is an observer $\mathrm{M}$ at the origin $\mathrm{O}$ of the $x$-axis of frame $S_{\mathrm{cl}}$, and $\mathrm{M}$ has a stop watch $\mathrm{W}$. In addition, there is an observer A at the origin $\mathrm{O}_{\mathrm{A}}$ of the $x_{\mathrm{A}}^{\prime}$-axis of frame $S_{\mathrm{A}}^{\prime}$, and $\mathrm{A}$ has a stop watch $\mathrm{W}_{\mathrm{A}}$.

Now, when rocket A passes in front of observer $\mathrm{M}$, observer $\mathrm{M}$ starts $\mathrm{W}$, and observer $\mathrm{A}$ starts $\mathrm{W}_{\mathrm{A}}$.

After that, when 1 (s) has elapsed on $\mathrm{W}$ in frame $S_{\mathrm{cl}}$, a light signal is emitted from frame $S_{\mathrm{cl}}$ toward frame $S_{\mathrm{A}}^{\prime}$. This light propagates isotropically from the light source.

According to the STR, an observer in frame $S_{\mathrm{cl}}$ finds the following relationship between the time $t$ which elapses on $\mathrm{W}$ and the time $t_{\mathrm{A}}^{\prime}$ which elapses on $\mathrm{W}_{\mathrm{A}}$.

$$
t_{\mathrm{A}}^{\prime}=\frac{t}{\gamma}=t\left(1-\frac{v^{2}}{c^{2}}\right)^{1 / 2} .
$$

Here, when 1 (s) is substituted for $t$,

$$
t_{\mathrm{A}}^{\prime}=0.8(\mathrm{~s})
$$

Here, this thought experiment is explained using Minkowski diagram (see Figure 3).

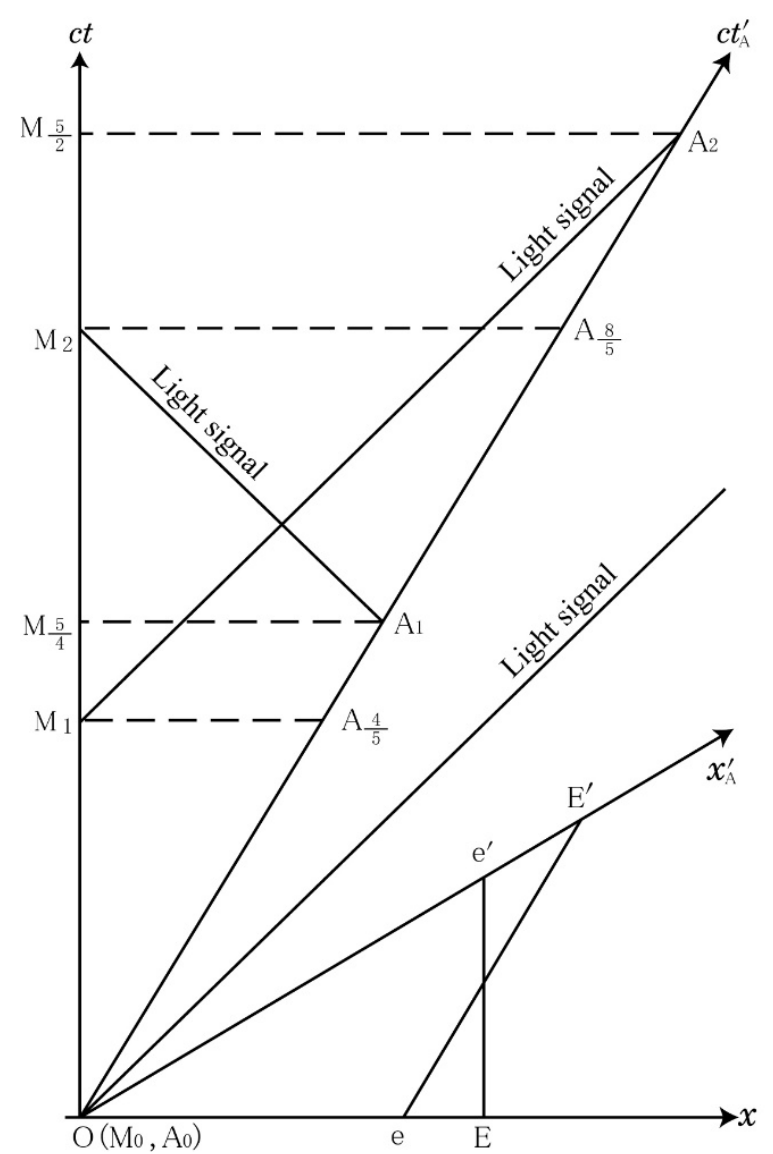

Figure 3. Minkowski diagram: This diagram corresponds to thought experiment 
Point $\mathrm{O}$ indicates both origins: $x=0, t=0$ and $x_{\mathrm{A}}^{\prime}=0, t_{\mathrm{A}}^{\prime}=0$. The point event $\mathrm{M}_{0}$ of the point light source $\mathrm{O}$ and the point event $\mathrm{A}_{0}$ of the point light source $\mathrm{O}_{\mathrm{A}}$ are at the origin $\mathrm{O}$. (Here, the subscripts " 0 " of the point events $\mathrm{M}_{0}$ and $\mathrm{A}_{0}$ mean, respectively, $t=0$ and $t_{\mathrm{A}}^{\prime}=0$.).

The $x$-axis indicates the $x$-axis of the inertial frame $S_{\mathrm{cl}}$ when $t=0$. In addition, the $x_{\mathrm{A}}^{\prime}$-axis indicates the $x_{\mathrm{A}}^{\prime}$ -axis of the inertial frame $S_{\mathrm{A}}^{\prime}$ when $t_{\mathrm{A}}^{\prime}=0$.

The $c t$-axis is the path for $x=0$. Put another way, it is the world line of the source $\mathrm{O}$. The $c t_{\mathrm{A}}^{\prime}$-axis is the world line of the source $\mathrm{O}_{\mathrm{A}}$.

In addition, the straight line extending at a $45^{\circ}$ angle from $\mathrm{O}$ indicates the light signal emitted from the two light sources at the instant that $\mathrm{O}$ and $\mathrm{O}_{\mathrm{A}}$ pass by each other (For details on this space-time diagram, see Reference) (Suto, 2016).

When $\mathrm{W}$ in frame $S_{\mathrm{cl}}$ is at 1 (s), a light signal is emitted from $\mathrm{O}$ to $\mathrm{O}_{\mathrm{A}}$. That light propagates isotropically with respect to $\mathrm{O}$. Then it arrives at $\mathrm{O}_{\mathrm{A}}$ when $\mathrm{W}_{\mathrm{A}}$ on rocket $\mathrm{A}$ is 2 (s). (This light signal corresponds to the world line $\mathrm{M}_{1} \mathrm{~A}_{2}$ ) (see Figure 4).
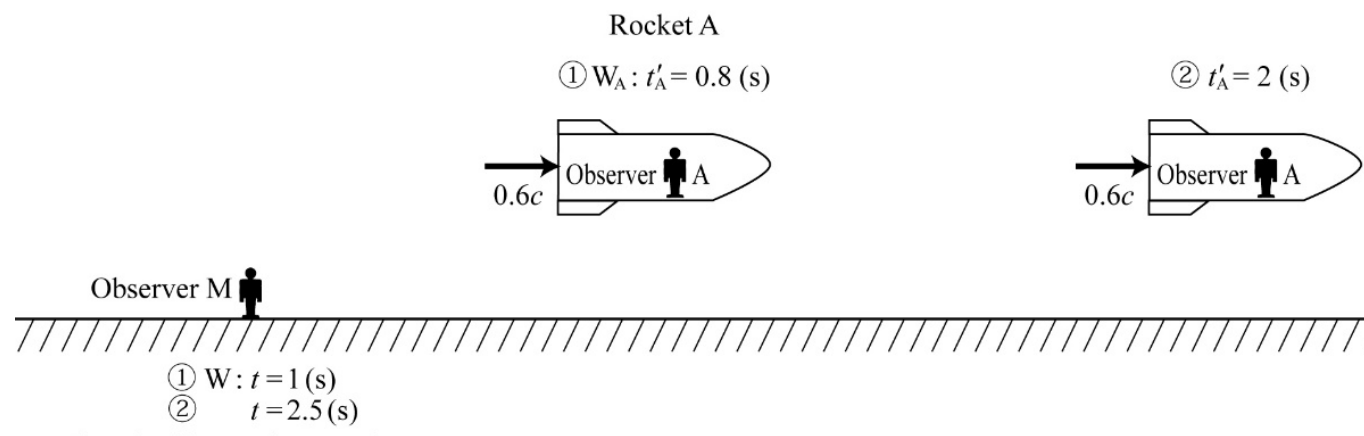

Classically stationary frame

Figure 4. Equation (22a), Explanation of world line $\mathrm{M}_{1} \mathrm{~A}_{2}$. Light emitted from light source $\mathrm{O}$ propagates isotropically. The distance traveled by the light signal becomes the distance between $\mathrm{O}$ and $\mathrm{O}_{\mathrm{A}}$ when light arrives at rocket $\mathrm{A}$

In the inverse case, when $\mathrm{W}_{\mathrm{A}}$ on rocket $\mathrm{A}$ is 1 (s), a light signal is emitted from $\mathrm{O}_{\mathrm{A}}$ to $\mathrm{O}$. That light arrives at $\mathrm{O}$ when $\mathrm{W}$ of frame $S_{\mathrm{cl}}$ is 2 (s) (This light signal corresponds to the world line $\mathrm{A}_{1} \mathrm{M}_{2}$ ).

These results show that symmetry exists between the two inertial frames. The following elucidates the mechanism whereby this symmetry holds.

In the case of this paper, where frame $S_{\mathrm{cl}}$ has been introduced, frame $S_{\mathrm{cl}}$ is always the stationary frame. Here, let us express the situation of the propagation of the light signals $\left(\mathrm{M}_{1} \mathrm{~A}_{2}\right.$ and $\left.\mathrm{A}_{1} \mathrm{M}_{2}\right)$ as follows.

$$
\begin{array}{lll}
\text { World line } & \mathrm{M}_{1} \mathrm{~A}_{2}: t=1(\mathrm{~s}) \rightarrow t_{\mathrm{A}}^{\prime}=2(\mathrm{~s}) . \\
\text { World line } & \mathrm{A}_{1} \mathrm{M}_{2}: t_{\mathrm{A}}^{\prime}=1(\mathrm{~s}) \rightarrow t=2(\mathrm{~s}) .
\end{array}
$$

Here the prime mark 'signifies a moving frame. From the perspective of this paper, frame $S_{\mathrm{cl}}$ is a rectangular coordinate system, and frame $S_{\mathrm{A}}^{\prime}$ is an oblique coordinate system. (In the STR, in contrast, rectangular and oblique coordinate systems are equivalent, and this is a tentative assumption.)

In contrast, in the STR which regards the two inertial frames as equivalent, the expression for Equation (22b) changes to the following:

$$
t_{\mathrm{A}}=1(\mathrm{~s}) \rightarrow t^{\prime}=2(\mathrm{~s})
$$

4.2 Light Propagation $M_{1} A_{2}$ as Viewed by an Observer in Frame $S_{\mathrm{cl}}$ (Equation (22a))

First, from Equation (20), the time $t_{\mathrm{A}}^{\prime}$ of $\mathrm{W}_{\mathrm{A}}$ is $0.8(\mathrm{~s})$ when the time $t$ of $\mathrm{W}$ is $1(\mathrm{~s})$.

Next, if $x$ is taken to be the distance which $\mathrm{O}_{\mathrm{A}}$ moves while 1 (s) elapses on $\mathrm{W}$ in frame $S_{\mathrm{cl}}$,

$$
x=v t=0.6 c \times 1=0.6 c(\mathrm{~m}) .
$$

Now, a light signal is emitted from $\mathrm{O}$ to $\mathrm{O}_{\mathrm{A}}$ when $t=1$ (s). If the time $t$ required for that light signal to reach $\mathrm{O}_{\mathrm{A}}$ is measured with $\mathrm{W}$ in frame $S_{\mathrm{cl}}$, then the following equation holds. 


$$
c t=0.6 c(1+t) .
$$

If this is used to find $t$,

$$
t=1.5 \text { (s). }
$$

Equation (20) is used to find the time $t_{\mathrm{A}}^{\prime}$ which elapses in frame $S_{\mathrm{A}}^{\prime}$ while 1.5 (s) passes in W. Here, if 1.5 is substituted for $t$ and $0.6 c$ for $v$ in Equation (20),

$$
t_{\mathrm{A}}^{\prime}=1.2(\mathrm{~s}) \text {. }
$$

Therefore, the time when the light signal emitted from $\mathrm{O}$ (when the time of $\mathrm{W}$ was $1(\mathrm{~s})$ ) arrives at $\mathrm{O}_{\mathrm{A}}$ is found by totaling (21) and (27). That is,

$$
t_{\mathrm{A}}^{\prime}=0.8+1.2=2(\mathrm{~s}) .
$$

The following time in the diagram corresponds to Equation (28).

$$
\text { Time } \mathrm{A}_{0} \mathrm{~A}_{2}=\text { Time } \mathrm{A}_{0} \mathrm{~A}_{4 / 5}+\text { Time } \mathrm{A}_{4 / 5} \mathrm{~A}_{2} \text {. }
$$

Up to this point, the predictions of this paper and the STR agree.

4.3 Light Propagation $A_{1} M_{2}$ Seen From Observer in Frame $S_{\mathrm{cl}}$. Explanation of this Paper (Equation (22b))

The light signal emitted from rocket $\mathrm{A}$ when $\mathrm{W}_{\mathrm{A}}$ on rocket $\mathrm{A}$ was 1 (s) arrives at $\mathrm{O}$ when $\mathrm{W}$ is 2 (s). This propagation situation $A_{1} M_{2}$ is interpreted as follows by observer $M$ (see Figure 5).
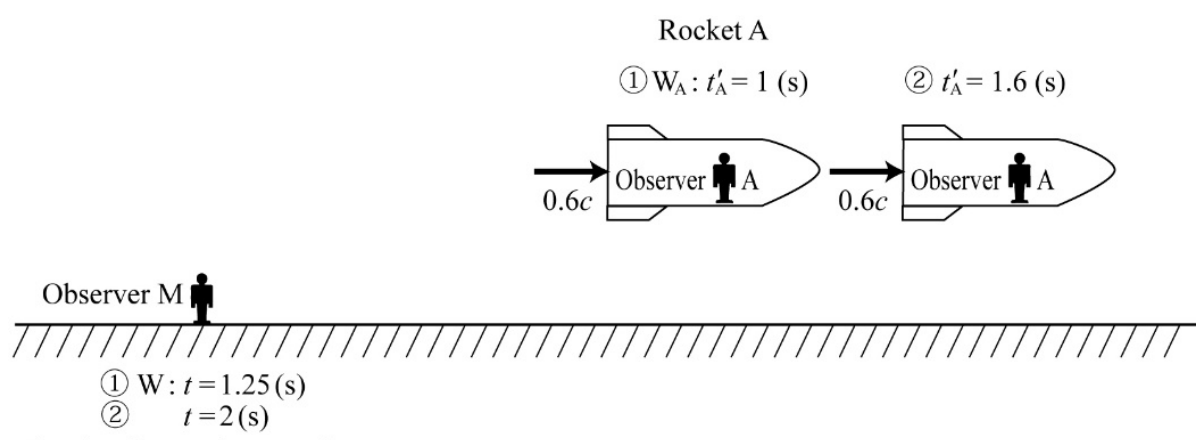

Classically stationary frame

Figure 5. Equation (22b), Explanation of world line $\mathrm{A}_{1} \mathrm{M}_{2}$. When observed from the frame $S_{\mathrm{cl}}$, the light signal emitted from rocket A when $t_{\mathrm{A}}^{\prime}=1(\mathrm{~s})$ arrives at frame $S_{\mathrm{cl}} 0.75(\mathrm{~s})$ later on the clock in frame $S_{\mathrm{cl}}$. The distance traveled by the light signal in this case becomes the distance between $\mathrm{O}$ and $\mathrm{O}_{\mathrm{A}}$ when light was emitted

\section{from rocket $\mathrm{A}$}

Interpretation 1: The observer M predicts the time $t$ which elapses on $\mathrm{W}$ when $1(\mathrm{~s})$ passes on $\mathrm{W}_{\mathrm{A}}$ as follows based on Equation (20).

$$
t=\frac{1}{\gamma}=1.25(\mathrm{~s})
$$

Now if $x$ is taken to be the distance $\mathrm{O}_{\mathrm{A}}$ moves while 1.25 (s) passes in the stationary frame,

$$
x=0.6 c \times 1.25=0.75 c(\mathrm{~m}) .
$$

The observer in frame $S_{\mathrm{cl}}$ applies the principle I to the propagation of the light signal emitted from $\mathrm{O}_{\mathrm{A}}$.

According to this principle, the light speed does not depend on the velocity of rocket A. Here, if the time required for the light signal to propagate over the distance $0.75 \mathrm{c}(\mathrm{m})$ is measured with $\mathrm{W}$ and taken to be $t$,

$$
t=0.75 c \div c=0.75(\mathrm{~s}) \text {. }
$$

Therefore, the time when light emitted from $\mathrm{O}_{\mathrm{A}}$ (when the time of $\mathrm{W}_{\mathrm{A}}$ was $1(\mathrm{~s})$ ) arrives at $\mathrm{O}$ can be found by totaling (30) and (32). That is,

$$
t=1.25+0.75=2(\mathrm{~s}) \text {. }
$$


The following time in the diagram corresponds to Equation (33).

$$
\text { Time } \mathrm{M}_{0} \mathrm{M}_{2}=\text { Time } \mathrm{M}_{0} \mathrm{M}_{5 / 4}+\text { Time } \mathrm{M}_{5 / 4} \mathrm{M}_{2} \text {. }
$$

4.4 Light Propagation $A_{1} M_{2}$, Equation (23). (Explanation of the STR)

\subsubsection{Explanation of Observer A in Rocket A}

Observer A assumes the principle of relativity, and regards the coordinate system of rocket A as a stationary frame. Also, the situation where a light signal is emitted and propagated is explained by Figure 6 not Figure 5 (see Figure 6). The observer A explains Equation (23) by using the logic employed by observer M (Equations (24) to (28)) as is.

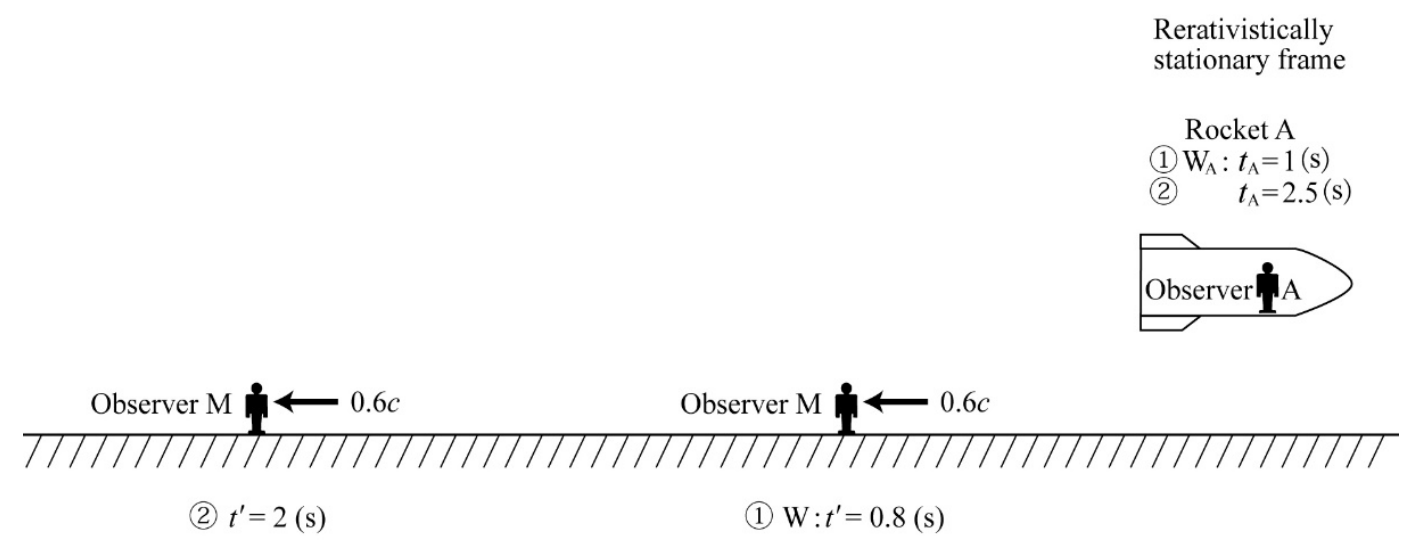

Figure 6. In Figure4, frame $S_{\mathrm{cl}}$ is a stationary frame, but in this figure the coordinate system of rocket A becomes the stationary frame. Light emitted from rocket A propagates isotropically (principle E)

\subsubsection{Explanation of Observer M}

Interpretation 2: Here, we will interpret Figure 6 from the standpoint of observer M. In the STR, it is possible for observers in all inertial frames to regard their own coordinate system as a stationary frame. In Figure 4, observer $\mathrm{M}$ predicts that $t_{\mathrm{A}}^{\prime}=0.8(\mathrm{~s})$ when $t=1(\mathrm{~s})$, and predicts that $t_{\mathrm{A}}^{\prime}=0.64(\mathrm{~s})$ when $t=0.8(\mathrm{~s})$. However, in Figure 6 the coordinate system for the observer M suddenly becomes a moving frame. At this time, observer $M$ must determine that, when his own clock reads $0.8(\mathrm{~s})$, the time in rocket $\mathrm{A}$ is not 0.64 (s) but rather 1 (s) (Problem 1).

The coordinate system of observer M which emits the light signal is a stationary frame, but the coordinate system of observer $\mathrm{M}$ who receives the light signal is a moving frame. This is extremely unnatural. In order for the observer M to justify the STR, he must regard the coordinate system of rocket A, which clearly cannot be said to be a stationary frame, as a stationary frame.

The observer M calculates the distance $x$ between the two $0.8(\mathrm{~s})$ after the rocket passes by in the following way.

$$
x=0.6 c \times 0.8=0.48 c(\mathrm{~m}) .
$$

The light signal emitted from rocket A when $t_{\mathrm{A}}=1(\mathrm{~s})$ propagates isotropically (principle E). Observer $\mathrm{M}$ is moving away from that light source at a speed of $0.6 c$. For observer $\mathrm{M}$ to find the speed of light $v$, he must not apply the velocity addition laws of the STR, and must instead calculate as follows (Problem 2).

$$
v=c-0.6 c=0.4 c \text {. }
$$

Observer M calculates the time $t^{\prime}$ which elapses on his own clock as follows.

$$
t^{\prime}=\frac{x}{v}=\frac{0.48 c}{0.4 c}=1.2(\mathrm{~s})
$$

From this, the time $t^{\prime}$ of $\mathrm{M}$ when the light signal arrives at observer $\mathrm{M}$ has the following value.

$$
t^{\prime}=0.8+1.2=2(\mathrm{~s}) \text {. }
$$

Finally, the light signal emitted from rocket A when $t_{\mathrm{A}}=1(\mathrm{~s})$ arrives at observer $\mathrm{M}$ when $t^{\prime}=2(\mathrm{~s})$. That is,

$$
t_{\mathrm{A}}=1(\mathrm{~s}) \rightarrow t^{\prime}=2(\mathrm{~s}) \text {. }
$$


To explain Equation (23), which is supported by the STR, observer M must use two types of logic (Problem 1 and Problem 2) which are unacceptable under the STR.

\section{Conclusion}

The results obtained thus far are summarized in the following table.

Table 1. Comparison of explanation in this paper (Figure 5) and explanation of the STR (Figure 6) for light signal propagation $\mathrm{A}_{1} \mathrm{M}_{2}$

\begin{tabular}{|c|c|c|}
\hline Explanation of world line $\mathrm{A}_{1} \mathrm{M}_{2}$ & Interpretation 1 & Interpretation 2 \\
\hline Interpreting observer & Observer $\mathrm{M}$ in stationary frame & Observer $\mathrm{M}$ in moving frame \\
\hline Applicable theory & This paper & STR \\
\hline Figure needed for explanation & Figure 5 & Figure 6 \\
\hline $\begin{array}{l}\text { Time on clock in rocket A when light signal is } \\
\text { emitted }\end{array}$ & $1(\mathrm{~s})$ & $1(\mathrm{~s})$ \\
\hline Time in $S_{\mathrm{cl}}$ when light signal is emitted & $1.25(\mathrm{~s})$ & $0.8(\mathrm{~s})$ \\
\hline $\begin{array}{l}\text { Time elapsed on clock of observer } M \text { during } \\
\text { propagation of light signal }\end{array}$ & $0.75(\mathrm{~s})$ & $1.2(\mathrm{~s})$ \\
\hline Time on clock when light signal arrives & $2(\mathrm{~s})$ & $2(\mathrm{~s})$ \\
\hline Light signal propagation process & Equation $(22 b)$ & Equation (23) \\
\hline $\begin{array}{l}\text { Principle for explaining propagation of light } \\
\text { signal }\end{array}$ & Principle of constancy of light speed I & Principle of constancy of light speed E \\
\hline Distance over which light signal propagates & $\begin{array}{l}\text { Distance between } 2 \text { light sources when } \\
\text { light signal is emitted }\end{array}$ & $\begin{array}{l}\text { Distance between } 2 \text { light sources when } \\
\text { light signal arrives }\end{array}$ \\
\hline Speed of light from perspective of observer M & $c$ & $0.4 c$ \\
\hline Symmetry between 2 coordinate systems & Does not hold & Holds \\
\hline Correctness of interpretation & Correct & Incorrect \\
\hline
\end{tabular}

In the STR, inertial frames $S$ and $S^{\prime}$ moving at constant speed relative to each other are treated as equivalent. Therefore, the following situation can naturally occur under the STR.

$$
\begin{aligned}
& \text { When } t=1(\mathrm{~s}), t_{\mathrm{A}}^{\prime}=0.8(\mathrm{~s}) . \\
& \text { When } t_{\mathrm{A}}=1(\mathrm{~s}), t^{\prime}=0.8(\mathrm{~s}) .
\end{aligned}
$$

However, the following relationships cannot both hold regarding an a priori rhythm.

$$
\begin{array}{ll}
\text { Prediction of observer M: } & \text { rhythm }(\mathrm{W}): \operatorname{rhythm}\left(\mathrm{W}_{\mathrm{A}}\right)=1: 0.8 . \\
\text { Prediction of observer A: } & \text { rhythm }\left(\mathrm{W}_{\mathrm{A}}\right): \operatorname{rhythm}(\mathrm{W})=1: 0.8 .
\end{array}
$$

With the STR, it is not possible to discuss rhythm, and thus the STR does not encounter the contradiction in (43). However, this paper discriminates between frames $S_{\mathrm{cl}}$ and $S_{\mathrm{cl}}^{\prime}$. Considered from the standpoint of realism, the clock in frame $S_{\mathrm{cl}}$ should run ahead of the clock in frame $S_{\mathrm{cl}}^{\prime}$.

Unlike classical physics, the STR is not a theory which searches for the values of actually existing physical quantities. It is also not a theory constructed with the aim of discovering physical laws.

Within the scope of section 4, the STR is mathematics for predicting the value of measured physical quantities based on the method designated by Einstein. The STR overlooked the existence of velocity vectors which may sometimes be attached to an inertial frame, and thus moved away from being physics describing the nature of reality as it is.

\section{Acknowledgments}

I would like to express my thanks to the staff at ACN Translation Services for their translation assistance. Also, I wish to express my gratitude to Mr. H. Shimada for drawing figures.

\section{Refferences}

Einstein A. (1923). The Principle of Relativity (pp. 38-41). New York: Dover Publication, Inc.

Suto, K. (2014). True Factors Determining the Ratio of Space Contraction and Time Dilation Predicted by the Special Theory of Relativity. Physics Essays, 27(4), 580-585. http://dx.doi.org/10.4006/0836-1398-27.4.580 
Suto, K. (2010). Violation of the special theory of relativity as proven by synchronization of clocks. Physics Essays, 23(3), 511-519. http://dx.doi.org/10.4006/1.3474836

Suto, K. (2015). Demonstration of the existence of a velocity vector missing from the special theory of relativity. Physics Essays, 28(3), 345-351. http://dx.doi.org/10.4006/0836-1398-28.3.345

Suto, K. (2017a). Method of Verifying the Existence of Velocity Vectors Excluded by the Special Theory of Relativity. Journal of Physical Mathematics, 4(4), 258-262. http://dx.doi.org/10.4172/2090-0902.1000258

Suto, K. (2017b). An Elucidation of the Symmetry of Length Contraction Predicted by the Special Theory of Relativity. Applied Physics Research, 9(3), 31-39. https://doi.org/10.5539/apr.v9n3p31

Suto, K. (2016). Elucidation of Time Symmetry Predicted by the Special Theory of Relativity. IOSR Journal of Applied Physics, 8(6), 20-27. http://dx.doi.org/10.9790/4861-0806042027

\section{Copyrights}

Copyright for this article is retained by the author(s), with first publication rights granted to the journal.

This is an open-access article distributed under the terms and conditions of the Creative Commons Attribution license (http://creativecommons.org/licenses/by/4.0/). 